\section{LAS GUERRAS EQUIVOCADAS. LA CIENCIA Y SU ENTORNO CULTURAL}

\author{
Sebastián Álvarez Toledo \\ Universidad de Salamanca \\ ORCID iD: http://orcid.org/0000-0003-1028-6316 \\ <sat@usal.es
}

Cómo citar este artículo/Citation: Álvarez Toledo, S. (2017). Las guerras equivocadas. La ciencia y su entorno cultural. Arbor, 193 (786): a423. doi: http://dx.doi.org/10.3989/ arbor.2017.786n4011

Recibido: 15 mayo 2016. Aceptado: 12 enero 2017.

RESUMEN: Han sido y son frecuentes los conflictos de la ciencia con su entorno cultural, en especial los que surgen de una supuesta incompatibilidad entre la cultura científica y la humanista. Un capitulo reciente de estos conflictos han sido las llamadas guerras de la ciencia, en las que se ha venido discutiendo sobre el lugar y prestigio social de la ciencia respecto de la cultura de humanidades y se han esbozado algunas propuestas para superar el tradicional antagonismo entre ellas. En este artículo trato de esta última cuestión y, retomando ideas de Otto Neurath acerca de la unidad de la ciencia, concluyo que el mejor modo posible de coexistencia y entendimiento entre ciencia y humanidades es el que tiene como base su colaboración en la solución de problemas sociales.

PALABRAS CLAVE: Las dos culturas; las guerras de la ciencia; E. O. Wilson; unidad de la ciencia; $O$. Neurath.

\section{THE WRONG WARS. SCIENCE AND ITS CULTURAL CONTEXT}

Copyright: (c) 2017 CSIC. Este es un artículo de acceso abierto distribuido bajo los términos de la licencia Creative Commons Attribution (CC BY) España 3.0.

ABSTRACT: Conflicts between science and its cultural context have been and are still frequent, especially those arising from an alleged incompatibility between scientific and humanistic cultures. A recent episode of these conflicts can be found in the so called science wars, in which the place and the social prestige of science with respect to humanistic culture have been discussed, and some proposals to overcome the traditional antagonism between them have been outlined. In this paper, I address this last matter and, returning to some ideas of Otto Neurath about the unity of science, I conclude that the coexistence and understanding between science and humanities should be based on their collaboration in the solution of social problems.

KEYWORDS: The two cultures; the science wars; E. O. Wilson; the unity of science; $\mathrm{O}$. Neurath. 


\section{INTRODUCCIÓN}

La cultura humana es un variado conjunto de conocimientos, creencias, valores, preferencias y habilidades que no poseemos por herencia genética sino que hemos adquirido por imitación y aprendizaje. Pero no todos los seres humanos compartimos unas mismas creencias, unas mismas opciones éticas, unas mismas preferencias estéticas, ni unas mismas capacidades, y la diversidad cultural ha dado lugar, a lo largo de la historia y en la actualidad, a conflictos y enfrentamientos, algunos más dramáticos que otros, entre sectores culturales o culturas que se han considerado incompatibles. Un área muy importante de la cultura humana es la cultura científica, que reúne disciplinas como las matemáticas, la física, la química, la biología o la economía, con sus correspondientes ramificaciones. Desde sus comienzos en la Europa renacentista, la ciencia moderna ha venido ganando prestigio social, y aunque actualmente la valoración de la ciencia dista mucho de la veneración acrítica que suscitó en un pasado reciente, y es general una actitud de sospecha y vigilancia respecto de sus aplicaciones tecnológicas y de los objetivos de muchas de sus investigaciones teóricas, no cabe duda de que la ciencia es una de las instituciones más valoradas socialmente y que más confianza suscita en todo el mundo.

Sin embargo, ha habido en los últimos siglos y surgen de vez en cuando en la actualidad múltiples conflictos o enfrentamientos entre la ciencia y su entorno cultural. Tal vez los más enconados son los que han tenido como motivo la incompatibilidad entre determinadas teorías científicas y concepciones del mundo basadas en creencias religiosas. Es inevitable mencionar en este aspecto la oposición de las iglesias cristianas a la naciente teoría heliocéntrica de Copérnico, tanto por parte del joven protestantismo como de la vieja ortodoxia católica. En 1539, antes de la publicación de De Revolutionibus, Lutero advertía:

\footnotetext{
"Algunos han prestado atención a un astrólogo advenedizo que se esfuerza en demostrar que es la Tierra la que gira y no el cielo o el firmamento, el sol y la luna [...] Este loco anhela trastocar por completo la ciencia de la astronomía, pero las Sagradas Escrituras nos enseñan (Josué 10,13) que Josué ordenó al sol, y no a la Tierra, que se parara".
}

También Melanchthon y Calvino combatieron las ideas de Copérnico esgrimiendo la verdad incontestable de la Biblia. Melanchthon consideraba "una falta de honestidad y de decencia mantener en público" la teoría heliocéntrica y que "un espíritu justo debe ad- mitir la verdad revelada por Dios y someterse a ella". Y en su Comentario al Génesis, Calvino se preguntaba: "¿quién osará colocar la autoridad de Copérnico por encima de la del Espíritu Santo?"1. Por otra parte y como es bien sabido, la astronomía copernicana, aunque utilizada por la iglesia católica ya en 1582 para reformar el calendario juliano, fue condenada por esta debido a que su estructura heliocéntrica se oponía a la imagen bíblica del universo. De ahí que se obligara a Galileo a renunciar a tal creencia y, a pesar de ello, se le condenara a arresto domiciliario en 1633 , en el que murió a los pocos años.

Desde la publicación de El origen de las especies, la teoría darwinista de la evolución ha sido objeto de variadas impugnaciones por parte de exaltados religiosos, defensores de la veracidad del relato bíblico de la creación. Por ejemplo, el Estado de Tennessee emprendió en 1925 una acción legal para prohibir su enseñanza, y los pleitos de este tipo han llegado a nuestros días en Estados Unidos con el creacionismo transmutado en una teoría pseudocientífica, la del diseño inteligente. Los partidarios de esta teoría argumentan que el evolucionismo darwiniano es insuficiente para dar razón de la complejidad de los seres vivos y que es necesario explicarla como resultado del plan trazado por una inteligencia sobrehumana, y exigen el reconocimiento de su teoría en la enseñanza pública estadounidense. Sobra decir que tales pretensiones se han encontrado con el rechazo de la comunidad científica y de los jueces. De todos modos, los conflictos entre las teorías científicas y los dogmas teológicos son, por lo general, cosas del pasado. Actualmente, al menos en occidente, las distintas confesiones religiosas han acabado admitiendo que no hay que interpretar literalmente los textos sagrados en sus ocasionales descripciones o explicaciones de los fenómenos naturales. Pero no siempre la oposición a los contenidos de las teorías científicas proceden de la religión: en la URSS de los años treinta y cuarenta, por ejemplo, el lysenkismo prohibió la investigación en líneas como el evolucionismo o la genética por su incompatibilidad con ciertos dogmas marxistas, como la transmisión de los caracteres adquiridos, y no faltaron los ataques a la teoría de la relatividad, a la mecánica cuántica y a cosmólogos sospechosos de haber contravenido las doctrinas del Anti-Düring de Engels.

\section{CUESTIÓN DE ESTILO}

Otros enfrentamientos han estado relacionados no con los contenidos del conocimiento científico, sino con su relevancia cultural o humana, y con el estilo, 
métodos y objetivos propios de la investigación científica. Encontramos este tipo de críticas ya en el humanismo renacentista. El renacimiento fue un movimiento cultural de amplio alcance que se caracterizaba por la ruptura con la tutela religiosa de la cultura propia de la Edad Media y la vuelta a los saberes de la cultura clásica grecolatina. Este ambiente de liberalización del pensamiento favoreció sin duda el nacimiento de la ciencia moderna. Sin embargo, el humanismo renacentista, cuya concepción antropocéntrica situaba al hombre por encima de la naturaleza y radicalmente separado de ella ${ }^{2}$, se sentía poco atraído por textos antiguos de carácter científico, como los de Tales de Mileto o Pitágoras, y de la cultura clásica prefería los escritos de teatro, poesía, gramática o retórica, que le acercaban a las tradicionales cuestiones filosóficas sobre el ser humano y le permitían un lenguaje más elocuente y un latín más elegante. Las siguientes palabras de Petrarca son muy significativas respecto del poco aprecio que muchos humanistas del renacimiento mostraban por el conocimiento y la explicación de los fenómenos naturales.

\footnotetext{
"Aunque todas estas cosas fueran verdaderas, no contribuirían en modo alguno a una vida feliz, pues ¿en qué nos ayuda familiarizarnos con la naturaleza de los animales, pájaros, peces y reptiles si seguimos ignorándolo todo respecto a la naturaleza de la especie humana a la que pertenecemos, y no sabemos o no nos preocupamos por saber, de dónde venimos y hacia dónde vamos?" (Capelli, 1906, pp. 24-25).
}

También encontramos este tipo de menosprecio de la ciencia por su supuesta irrelevancia cultural en la crítica romántica al programa racionalista de la llustración. E independientemente de que se pueda calificar a Nietzsche como un filósofo romántico anti-ilustrado o simplemente como un ilustrado crítico, sus opiniones sobre la ciencia intentan una descalificación de esta por su estilo de pensamiento y la naturaleza de sus resultados. En realidad, la crítica nietzscheana de la ciencia se deriva de su valoración negativa del conocimiento conceptual respecto del conocimiento intuitivo y las impresiones directas, espontáneas y fugaces de lo individual e irrepetible. Para Nietzsche, los conceptos son creaciones humanas que resultan de la búsqueda de lo general y permanente, de la anulación de las diferencias, y se convierten en "metáforas de las cosas que no corresponden en absoluto a las esencias primitivas"; son, en fin, toscas representaciones, descoloridas y petrificadas, de las intuiciones originales. Según Nietzsche, el conocimiento conceptual, condición de la racionalidad humana, "insufla en la lógica el rigor y la frialdad peculiares de la matemática" y tiene su mejor expresión en la formulación de las leyes naturales, que conforman el contenido de la ciencia moderna. Tales leyes, "incomprensibles en su esencia", se limitan a recoger relaciones superficiales entre fenómenos, a las que otorgan una validez espaciotemporal ilimitada que no es un descubrimiento, sino una concesión, un presupuesto, de modo que, por ejemplo, "toda la regularidad de las órbitas de los astros y de los procesos químicos, regularidad que tanto respeto nos infunde, coincide en el fondo con aquellas propiedades que nosotros introducimos en las cosas" (Nietzsche, 1873/1980, pp. 8-16). Resulta curioso cómo la teoría de los conceptos de Nietzsche, a partir del supuesto difícilmente rebatible de que los conceptos son abstracciones o construcciones humanas que recogen ciertas semejanzas entre objetos o sucesos, desemboca gratuitamente en una visión claramente despectiva de la racionalidad humana y de la ciencia; una conclusión en la que juega un papel decisivo su permanente añoranza del conocimiento de las "esencias" de las cosas y los fenómenos.

Esta depreciación de la ciencia por su supuesta irrelevancia cultural la encontramos también, aunque con matices propios, en Heidegger, quien en su Introducción a la metafísica, tras defender la primacía de la filosofía sobre la ciencia tanto "lógicamente" como en cuanto a "la existencia espiritual", cree detectar en la ciencia, ya sea en su aspecto práctico o tecnológico como en su aspecto teórico, un alarmante "debilitamiento del espíritu", e incluso se atreve a denunciar que la interpretación de la ciencia "como valor cultural, trata de encubrir la impotencia del espíritu por medio de una inconsciente tendencia a la mentira" (Heidegger,1953/2001, pp. 22-54).

Ya sea por lejana influencia del humanismo renacentista o por la más cercana del romanticismo, el caso es que hasta no hace mucho, y todavía en parte, la admiración por los prodigios del desarrollo científico y sus aplicaciones técnicas ha convivido en occidente con la convicción de que la verdadera cultura o lo más valioso de ella (esto es, lo más excelente, profundo y refinado) se situaba en el terreno de las humanidades, es decir, de la literatura, el teatro, la poesía, el arte, la historia, la filosofía, el cine, etc. Aunque se reconocieran los méritos del estudio de la naturaleza y sus secretos, mucho más interesante se ha venido considerando el afán por desentrañar los misterios de la condición humana, individual, social o histórica, dando por supuesto que se trata de dos territorios incomunicados. Esta situación es la que 
propicia la última etapa de los conflictos entre la ciencia y su entorno cultural que vengo mencionando: una etapa en la que no se discute sobre la verdad o la relevancia de los contenidos de la ciencia ni sobre la bondad de sus aplicaciones, sino sobre el prestigio social de la ciencia y las humanidades y el reconocimiento por parte de las instituciones y de los ciudadanos, al tiempo que se esbozan algunas propuestas para superar la zanja entre estas dos áreas culturales. Cabe situar el inicio de esta tercera fase en la ya célebre conferencia de C. P. Snow en la Universidad de Cambridge en 1959, "The two Cultures", publicada posteriormente y ampliada en 1964.

La expresión de "las dos culturas" alude claramente a la tradicional división entre la cultura científica y la de las humanidades. Sin embargo Snow se refiere solo a las diferencias e incompatibilidades que cree advertir entre los físicos y los "intelectuales literarios" (novelistas, poetas, dramaturgos), entendiendo que los físicos son los científicos más representativos, y que los literatos representan cualificadamente a la otra cara de la cultura, de modo que la contraposición entre físicos y literatos constituiría "una metáfora vistosa" (Snow, 1964/1977, p. 19) de la escisión de la cultura humana en dos mundos incomunicados y muy distintos psicológica, intelectual y moralmente. En tal contraposición los científicos aparecen como ciudadanos solidarios, tenaces y optimistas, convencidos de que pueden hacer mucho por resolver los grandes problemas de la humanidad. Son, según Snow, personas inteligentes y acostumbradas al diálogo y el debate y "el grupo de intelectuales más sano que tenemos" en lo moral; y en cuanto a los resultados de su trabajo, los científicos crean una cultura "rica en contenido teórico, generalmente mucho más rigurosa y casi siempre de un nivel conceptual más alto que las teorías de los intelectuales literarios" (Snow, 1964/1977, p. 12, p. 17, pp. 22-23). Estos, sin embargo, gozarían de un prestigio y una influencia social muy superior a los que se otorgan a los científicos, aunque viven apegados a la cultura tradicional, desconectados del conocimiento científico y ajenos o reticentes a las innovaciones tecnológicas y son, por lo general, seres antisociales e incluso reaccionarios ética y políticamente. Y como prueba de ello esgrime Snow las convicciones fascistas de Pound y Yeats, la actitud antisemita y belicista de Dostoievski o lo que presenta como pesimismo anti-tecnológico de Lawrence u Orwell. Snow entreveía, sin embargo, una superación de la división entre las dos culturas en una "tercera cultura" aún en ciernes, que estaría formada por las ciencias sociales: historia social, sociología, demografía, economía, psicología (Snow, 1964/1977, p. 72, pp. 80-82).

Si atendemos solo al tono maniqueo, simplista y tendencioso de este panfleto de Snow resulta inexplicable el eco que ha alcanzado posteriormente; sin embargo, hay que reconocer que tuvo el acierto y la oportunidad de llamar la atención sobre el hecho ya mencionado de que se llegara a identificar "la cultura" (o lo más excelente de la cultura humana) con la cultura humanista ${ }^{3} y$ denunciar que el término "intelectual" quedara reservado, por lo general, para profesionales de este área de la cultura y que muchos de ellos gozaran de un reconocimiento social excepcional y de una cercanía a los poderes públicos y centros de decisión de los que, por lo general, carecían los científicos.

Sin embargo, el panorama ha cambiado notablemente. Desde mediados del siglo $X X$ ha venido creciendo en la población la necesidad de una cultura científica básica, con la correspondiente oferta divulgativa por parte de muchos científicos, convencidos de que esta labor constituye una parte importante de su trabajo. Actualmente la información sobre ciencia y tecnología está presente en prensa, radio, televisión, librerías e internet, y son muchos los científicos (pensemos en Isaac Asimov, Stephen Hawking, Carl Sagan, Stephen Jay Gould, Richard Dawkins o Daniel Dennett) que han conseguido una imagen pública tradicionalmente reservada a muy pocos de ellos. Es un hecho que el prestigio social de la ciencia ha crecido considerablemente y que poca gente consideraría ya que se puede ser "culto" desconociendo conceptos básicos de la biología, la física o la tecnología.

Sin embargo, no han cesado en las últimas décadas los enfrentamientos entre "las dos culturas", si bien se ha tratado tan solo de una serie de reproches mutuos y de polémicas con poco calado conceptual y un discreto eco editorial entre determinados sociólogos y filósofos, por una parte, y un reducido número de científicos, por otra. Resulta desproporcionado que a estas escaramuzas dialécticas se les haya llamado "las guerras de la ciencia". De todos modos, me detendré brevemente en estas discusiones atendiendo a que uno y otro bando presentan sus puntos de vista como fórmulas para crear puentes o disolver fronteras entre la cultura científica y la humanista y poner fin a los tradicionales conflictos entre ellas.

\section{RELATIVISMO Y REDUCCIÓN}

La filosofía de los años sesenta rompió con la imagen tradicional de la ciencia, heredera de una pers- 
pectiva positivista. Autores como Popper, Hanson o Toulmin insistieron en el carácter hipotético y provisional del conocimiento científico y en la inevitable carga teórica de la observación y la experimentación en la ciencia, y como es bien sabido, Kuhn defendía que no se pueden comprender los cambios en el desarrollo de la ciencia atendiendo solo a criterios lógicos y empíricos y prescindiendo de factores externos (sociales, económicos, psicológicos, etc.). Sin embargo, esta oportuna labor crítica tuvo en el campo de la filosofía algunas derivaciones extremas que ofrecían una imagen irreconocible de la ciencia. Feyerabend llegó a sostener que dentro de una ciencia existen puntos de vista "a menudo tan diferentes como las ideologías subyacentes a las distintas culturas", que no existe un método científico específico y que "la ciencia es mucho más semejante al mito de lo que cualquier filosofía científica está dispuesta a reconocer" (Feyerabend, 1975/1981, p. 269, p. 289).

El giro sociológico que Kuhn, entre otros, introdujo en el análisis del desarrollo de la ciencia estuvo acompañado del incremento de interesantes estudios históricos, sociales, económicos y políticos sobre la ciencia. Y hubo sociólogos y filósofos que, desde una perspectiva similar a la de Feyerabend, intentaron despojar drásticamente a la imagen de la ciencia de atributos como la objetividad, la neutralidad, la autonomía, la universalidad o la racionalidad, de modo que, convertido en un producto cultural como cualquier otro, el conocimiento científico sería mero producto de las condiciones sociales, económicas o políticas del entorno en que surge, su análisis no exigiría categorías o criterios sustancialmente distintos a los utilizados en los estudios culturales de actividades como el cine, la religión o la literatura, y la misma actividad científica podría estar tan sujeta a la opinión pública como puede estarlo una determinada política educativa, fiscal o laboral. Sociólogos del conocimiento como Barnes, Bloor, Collins, Mackenzie o Pickering, para quienes el conocimiento no es más que un conjunto de creencias compartidas por determinados grupos o culturas, han defendido que los estudios sobre el conocimiento científico deben prescindir de consideraciones acerca de su verdad o su falsedad, su justificación o falta de ella, dado que los contenidos de estos criterios no tienen carácter universal, sino que varían según cada grupo social o cultural (Barnes y Bloor, 1982). Otros sociólogos (Knorr-Cettina, Latour, Woolgar), más centrados en el análisis de prácticas científicas concretas, han insistido en que los datos experimentales no son meros registros de fenómenos naturales, sino resultados de los acuerdos con los que se resuelven las controversias en el interior de los grupos de investigadores (Latour, 1987, p. 258); de modo que la investigación experimental no descubre hechos naturales ni formula verdades acerca de ellos, sino que construye los hechos, aunque luego legitime esos acuerdos y creaciones con la habitual retórica sobre la cuidadosa observancia del método científico (Latour y Woolgar, 1979, p. 228, pp. 236-237). Por su parte, el crítico cultural Aronowitz (1988), insistiendo en la fundamental semejanza de las ciencias con otras disciplinas y actividades culturales, ${ }^{4}$ afirmaba que también la ciencia de cada momento histórico y cada país es producto y reflejo de la ideología política dominante; de modo similar la psicóloga y filósofa Irigaray ha sostenido que "la ciencia hace siempre determinadas elecciones, determinadas exclusiones, debido sobre todo al sexo de los estudiosos que se dedican a ella" (Irigaray, 1987, p. 219); y la antropóloga Harding ha denunciado que la ciencia actual "no sólo es sexista, sino también racista, clasista y culturalmente coactiva" y que "la física, la química, las matemáticas y la lógica conservan las huellas de sus creadores culturales no menos que la antropología y la historia", a lo que añadía que el androcentrismo de la ciencia moderna, unido a la creencia en el carácter progresivo de la racionalidad científica, nos ha llevado a un punto en el que es necesaria "una revolución intelectual, moral, social y política más radical de lo que los fundadores de las modernas culturas occidentales jamás habrían imaginado" (Harding, 1986/2010, p. 9, p. 19). En este marco de revisión crítica de la ciencia como producto cultural y de propuestas de cambios en ella se inscriben los consejos del sociólogo Ross, que cree que la ciencia debería revisar sus pretensiones de validez universal y relativizar sus exigencias metodológicas a fin de que sean posibles modos distintos de hacer ciencia en los que se respete la diversidad de los valores culturales (Ross, 1996).

Tiene razón Hacking al afirmar que lo que estos constructivistas sociales combaten "no es la verdad de la proposiciones aceptadas por la ciencia, sino una imagen demasiado ensalzada de lo que hace la ciencia o la autoridad que los científicos reclaman para el trabajo que llevan a cabo" con el fin de "asegurar la autoridad cultural de la ciencia" (Hacking, 1999/2001, pp. 159-160). Y el modo de hacerlo consiste básicamente en democratizarla, pero en el sentido de despojarla de sus características propias hasta convertirla en una variedad cultural como cualquier otra, considerando que con ello contribuyen a superar la brecha entre ciencia y humanidades construyendo puentes entre ellas (Latour, 1999, p. 17). 
Como era de esperar, esta visión reduccionista de la ciencia suscitó diferentes respuestas desde el lado de la cultura científica. Por esos años aparecieron numerosos artículos y libros en los que, frente a tanta interpretación relativista, se reivindicaban los valores epistémicos en la ciencia como explicación de sus éxitos teóricos y prácticos ${ }^{5}$. En este apartado es inevitable mencionar el conocido e ingenioso "asunto Sokal". En 1996, el físico de la universidad de Nueva York Alan Sokal tuvo la perspicaz idea de enviar a la revista de sociología Social Text un artículo que era solo una leve exageración de afirmaciones relativistas respecto a la ciencia como las que acabamos de citar. En él encontramos despropósitos como que la gravedad cuántica (una teoría aún en ciernes) es un tipo de física liberadora que serviría de apoyo a las causas políticas progresistas; que la metodología de la ciencia posmoderna ofrece una valiosa herramienta intelectual al proyecto político de derribar las fronteras en la vida social y cultural; que es necesaria una matemática "emancipada" acorde con la nueva física posmoderna; o que la teoría del campo cuántico confirma el punto de vista de Lacan sobre la neurosis (Sokal, 1996). El artículo fue aceptado y poco más tarde Sokal desveló que se trataba de un artículo carente de rigor conceptual, plagado de absurdos y barbaridades bien trabados, cuya aceptación y publicación ponían de manifiesto la falta de criterio o la permisividad de la revista con los contenidos acordes con su ideología.

Hubo también respuestas indignadas de científicos a esa versión "posmoderna" de la ciencia, pero con un tono polémico diferente. Así el biólogo Paul Gross y el matemático Norman Levitt, aunque llevan a cabo una crítica certera de la frivolidad e incompetencia con que algunos sociólogos y críticos literarios opinan sobre la actividad científica, mantienen una actitud sarcástica y despectiva, y en el capítulo final llegan a sostener que, en contraste con el desconocimiento de la cultura científica que muestran muchos profesores de humanidades, los científicos están, por lo general, muy capacitados para enseñar historia o filosofía de forma competente (Gross y Levitt, 1994). En un tono muy semejante y de un modo no menos gratuito Hawking y Mlodinow (2010, p. 11) afirman que la filosofía, debido a su desconexión con la física actual, ya no es capaz de ofrecer respuestas a las viejas preguntas que le son propias y que son los científicos quienes tienen que retomar esa tarea.

Esta idea de un relevo en las tareas que han estado tradicionalmente en manos de intelectuales de humanidades es visible en la reacción de algunos cien- tíficos a los excesos críticos de estos y está presente, por ejemplo, en el planteamiento editorial del libro colectivo La tercera cultura. Este libro, recopilado por el agente literario John Brockman, ofrece una serie de interesantes artículos divulgativos sobre física, genética, biología evolutiva, informática, neurofisiología, física, etc. a cargo de reconocidos especialistas (como Gell-mann, Penrose o Dawkins). Pero en la introducción Brockman aclara que el término de tercera cultura del título se refiere a los trabajos divulgativos de científicos que "están ocupando el lugar del intelectual clásico a la hora de poner de manifiesto el sentido más profundo de nuestra vida, replanteándonos quiénes y qué somos" (Brockman, 1995, p. 13). En términos parecidos se expresan varios autores del libro en esa misma introducción.

Esta aspiración de superar la tradicional escisión entre "las dos culturas" mediante la ocupación por parte de la cultura científica de terrenos que tradicionalmente han pertenecido a las humanidades, es decir, de unificar la cultura mediante una reducción a partir de los conocimientos que la ciencia nos ofrece o puede previsiblemente llegar a proporcionarnos, es central en el polémico libro del entomólogo Edward O. Wilson Consilience. La unidad del conocimiento. Las ideas de Wilson acerca de la unidad o unificación del conocimiento humano no siempre son coherentes. En su versión más razonable tal unificación implicaría, por ejemplo, que todo proceso mental "tiene un fundamento físico y es consistente con las ciencias naturales" o que ciertas predisposiciones naturales influyen eficazmente en determinadas manifestaciones o instituciones culturales, como el totemismo o las ceremonias religiosas (Wilson, 1999, p. 143, pp. 245247). Sin embargo, en el último capítulo Wilson reclama una interpretación decididamente reduccionista de sus consideraciones acerca de la unidad del conocimiento y dice haber argumentado que, aunque existan diferentes explicaciones de los fenómenos (explicaciones antropológicas, psicológicas, etc.), solo hay "intrínsecamente" un tipo de explicación, porque "todos los fenómenos tangibles, desde el nacimiento de las estrellas hasta el nacimiento de las instituciones sociales se basan en procesos materiales que en último término son reducibles, por largas y tortuosas que sean las consecuencias, a las leyes de la física". De modo que las peculiaridades de nuestra especie y, en general, la cultura humana "sólo tendrán sentido completo cuando se conecten mediante explicaciones causales a las ciencias naturales" (Wilson, 1999, pp. 389-390).

Hemos visto que tanto la crítica relativista de la ciencia como la reacción de muchos defensores de la 
cultura científica tienen en común el afán de superar la brecha entre ciencia y humanidades mediante una unificación que adopta una forma distinta en cada caso: en el primero, esta unificación exigiría la disolución de las fronteras, esto es, la negación de cualquier demarcación de la ciencia; y en el segundo, la solución estaría en la reducción de la cultura humanista a cultura científica. No voy a detenerme a mostrar lo insatisfactorio de ambas propuestas, sino que me centraré en una cuestión que las ideas de Wilson suscitan. Tras conocer su propuesta es sensato preguntarse: ¿acaso la ciencia ha logrado una unificación de sus diversas ramas y especialidades como la que se propone en el caso de "las dos culturas"?, ¿en qué consiste o podría llegar consistir esa unidad de la ciencia? En lo que sigue partiré de estas cuestiones con el fin de extraer algunas consecuencias que puedan ser aplicables a los enfrentamientos o supuestas incompatibilidades entre la cultura científica y la humanista.

\section{LA UNIDAD DE LA CIENCIA}

Uno de los grandes valores en el desarrollo de la ciencia es la unificación, el descubrimiento de que fenómenos, leyes o teorías aparentemente distintos responden a unos mismos principios, de modo que elementos teóricos antes dispersos pasan a formar parte de una teoría más general capaz de dar razón de cada uno de ellos. De hecho, en la ciencia moderna, la física newtoniana surge como unificación, mediante el concepto de fuerza gravitatoria, de los descubrimientos de Copérnico y Kepler en mecánica celeste y de Galileo en la caída de los cuerpos en la Tierra. Luego en el siglo XIX, tanto el electromagnetismo de Maxwell, que aglutinó en una misma teoría la luz, el calor radiante, la electricidad y el magnetismo, como la teoría cinética del calor supusieron dos importantes logros unificadores de fenómenos diferentes en apariencia. Y en la segunda mitad del siglo XX la unificación de la fuerza electromagnética con las interacciones nucleares débil y fuerte reavivó la idea de una física plenamente unificada en lo que ha llegado a denominarse de modo grandilocuente la teoría del todo. Pero la física no es la única ciencia con éxito en este aspecto; ahora también se invocan profundas conexiones entre la química orgánica y la genética en el campo de la biología molecular.

No es extraño, por tanto, que la unidad de la ciencia haya sido uno de los grandes temas de la reflexión filosófica sobre esta. Podemos encontrar muestras de ello tanto en Kant como Comte y, sobre todo, en el Círculo de Viena, en cuyo documento fundacional,
"La concepción científica del mundo", se afirma que el grupo tiene como meta "lograr la ciencia unificada" (Carnap, Hahn, y Neurath, 1929). El modo más habitual (no el único, como veremos) de concebir esta labor unificadora de la filosofía tenía en Carnap su mejor exponente y consistía básicamente en dos tipos de reducción, una semántica y otra metodológica. La reducción semántica exigía disponer de un lenguaje de observación neutro teóricamente y seguro epistémicamente desde el que poder interpretar los distintos términos científicos, para descartar así injerencias metafísicas en el lenguaje de las teorías. Y la reducción metodológica, inspirada en la labor de Hilbert en la axiomatización de la geometría, estaba orientada a presentar la ciencia en su totalidad como un sistema jerárquico de teorías y leyes de diferentes niveles relacionados deductivamente a partir de los principios o leyes fundamentales de la física (Carnap, 1928). Este programa reduccionista no era ajeno a la convicción de que la naturaleza misma está organizada de tal modo que los principios de la física explican todo tipo de fenómenos y regularidades químicas, biológicas, mentales o sociales.

Sin embargo, a pesar de los mencionados logros en la unificación de teorías, la historia de la ciencia no nos permite ser muy optimistas respecto de la consecución de una ciencia unificada. Ciertamente, la teoría cinética del calor convirtió a la termodinámica en una teoría fenomenológica explicada básicamente desde la mecánica clásica, pero aún sigue pendiente de explicación la omnipresente irreversibilidad que el principio de entropía contempla; y en cuanto a la unificación de las fuerzas fundamentales, la fuerza gravitatoria se resiste a mostrarse compatible con las interacciones nucleares, cavando una zanja entre la relatividad general y la mecánica cuántica. Por otra parte, en biología, la perspectiva reduccionista de, por ejemplo, el genetista Watson o el entomólogo ya mencionado Wilson se ha encontrado con el escepticismo de Mayr o Levontin, quienes, frente al enfoque exclusivamente atomista en genética, han abogado por un acercamiento más holista y medioambiental. Finalmente, las ciencias cognitivas y las sociales, dada su diversidad de enfoques, propia de ciencias jóvenes que, además, se enfrentan a fenómenos altamente complejos, no prometen de momento una unificación interna.

Esta realidad ha tenido eco en la filosofía de la ciencia de las últimas décadas, que ha incluido en su agenda una revisión del viejo ideal de una ciencia unificada. Destaca en este aspecto la llamada Escuela 
de Stanford, que reúne a filósofos como John Dupré, Ian Hacking, Peter Galison, Patrick Suppes o Nancy Cartwright, entre otros. Según Cartwright, una malinterpretación de la naturaleza de las teorías científicas junto a una especie de realismo extremo en cuanto a su estructura han creado falsas expectativas respecto de la unificación de la ciencia. El éxito de muchas teorías científicas y de algunas unificaciones y reducciones teóricas parciales ha suscitado la creencia en un mundo racionalmente ordenado, en el que cada ley o regularidad empírica es una realización de un principio o ley fundamental, y que puede ser representado por un sistema teórico universal. Éste "anhelo de sistema" (Cartwright, 1999, p. 17), que ha acompañado a la física moderna durante siglos, estaba también presente en el proyecto de una ciencia unificada del Círculo de Viena y del empirismo lógico y lo está en el concepto de teoría del todo de la física contemporánea. Sin embargo, hay que tener en cuenta que una teoría científica (Cartwright se refiere especialmente a las teorías de la física) es solo una organización de leyes experimentales o fenomenológicas a partir de unos principios sumamente abstractos que no añaden ningún contenido a las leyes que articulan. $Y$ estas leyes fenomenológicas, que son las que describen el mundo, están circunscritas a situaciones concretas, son diversas y en general independientes entre sí. Incluso aquellas que tratan de un mismo tipo de fenómenos no forman de suyo una pirámide, sino un mosaico (patchwork). Si unimos estas consideraciones al hecho de que incluso las mejores teorías tienen un alcance limitado, dado que solo son aplicables a situaciones parecidas a las de sus modelos, es preciso concluir que lo que la ciencia actual nos muestra (y la física es buen ejemplo de ello) no es una unidad o la prueba de que esta es posible, sino una gran diversidad: una variedad de disciplinas con diferentes grados de precisión que tratan de campos distintos con algún que otro solapamiento errático. Por tanto, no hay razones para pensar que nuestro mundo está racionalmente estructurado de tal modo que cada suceso o regularidad obedezca a una ley fundamental. Lo que el conocimiento científico nos muestra es un mundo moteado ( $a$ dappled world): un mundo rico en cosas distintas con naturalezas diferentes que se comportan de modos diferentes, un mundo en el que muchos sucesos no están cubiertos por ninguna ley porque ocurren en la intersección de teorías donde no hay ninguna ley específica. Por tanto, la idea de la unidad de la ciencia como meta o ideal tiene como base un malentendido, fruto de la confusión entre las características de nuestras teorías y la propia naturaleza (Cartwright, 1999, pp.1-17).
En su defensa del pluralismo en la ciencia, Cartwright y otros autores (Cartwright, Cat, Flech y Uebel, 1996) han destacado la figura de Neurath, un autor notablemente comprometido con el proyecto de unificación de la ciencia, mostrando su cercanía al modo en que este concebía las relaciones entre las diversas disciplinas científicas. El sociólogo, economista y filósofo Otto Neurath fue uno de los padres del Círculo de Viena, creó el Instituto Internacional de la Ciencia Unificada en 1934 en La Haya, y en 1938 fundó en Chicago, junto con Carnap y Morris, la Enciclopedia Internacional de la Ciencia Unificada. Su concepción de la unidad de la ciencia, diferente, como veremos, de la habitual en el Círculo de Viena y sus epígonos, era acorde con su pensamiento político, su interés en la vida social y el bienestar de los ciudadanos ${ }^{6}$ y sus ideas sobre la función de la ciencia en la sociedad. La ciencia era para Neurath una empresa fundamentalmente social, cuyas leyes permiten la predicción y el control de fenómenos naturales y sociales y, por tanto, la solución de múltiples problemas (económicos, bélicos, educativos, de convivencia, etc.) que afectan a los ciudadanos. Sin embargo las leyes científicas son enunciados abstractos que se ocupan selectivamente de determinadas clases de fenómenos (físicos, químicos, biológicos o sociales) y sólo se cumplen apropiadamente en condiciones privilegiadas, diseñadas y producidas artificialmente en el laboratorio: unas condiciones muy distintas de las que espontáneamente se dan en la naturaleza o en la sociedad, donde los fenómenos son singulares, irrepetibles y sumamente complejos en cuanto a sus propiedades y sus causas, de modo que no se pueden predecir a partir de un solo tipo de leyes y exigen la utilización de leyes de distintas disciplinas:

"Es cierto que se pueden distinguir diferentes clases de leyes: por ejemplo, leyes químicas, biológicas o sociológicas; sin embargo, no se puede decir que la predicción de un proceso individual concreto dependa sólo de una clase concreta de leyes. Por ejemplo, el incendio de un bosque en un determinado lugar de la Tierra depende tanto del tiempo meteorológico como de si ha habido o no intervención humana. Sin embargo, esta intervención sólo se puede predecir si se conocen las leyes del comportamiento humano. Esto es, en ciertas circunstancias debe ser posible conectar todo tipo de leyes unas con otras" (Neurath, 1931/1983, p. 59, cursivas en el original).

Por tanto, la predicción y el control de fenómenos naturales y la solución de los problemas sociales exigen disponer de conocimientos y leyes provenientes de ciencias muy distintas. En esta consideración se 
basa el proyecto de Neurath de una ciencia unificada, que consistiría básicamente en hacer asequibles los conocimientos provenientes de diferentes ciencias para facilitar la colaboración y el entendimiento de científicos de distintas disciplinas en la solución de problemas sociales concretos: "la acción común nos empuja hacia una ciencia unificada" (Neurath, 1931/1973, p. 407). Pero tal proyecto no consistía en articular las distintas leyes y teorías científicas en un único sistema deductivo a partir de las leyes fundamentales de la física, como proponía, entre otros, Carnap, sino en una tarea enciclopédica (no muy distinta de la de L'Enciclopedie de Diderot) cuyo objetivo era ofrecer un "mosaico" o "agregado" de leyes de ciencias diferentes, una "orquestación interdisciplinar" del conocimiento científico en su rica variedad (Neurath, $1936 / 1983$ y $1946 / 1983$ ) o, como dice en otro lugar, "el stock de todas las leyes conectables y lógicamente y realmente compatibles" (Neurath, 1931/1973, p. 329). En definitiva, para Neurath, la creación de una ciencia unificada no era una cuestión de reducción teórica entre ciencias sino una tarea académica orientada a "servir con acierto a toda actividad transformadora" (Neurath, 1930/1983, p. 42).

Un necesario complemento de este proyecto era la formación de un lenguaje básico común que permitiera la comunicación entre científicos de distintas especialidades y de estos con el resto de ciudadanos. Sin embargo, Neurath no creía que para este fin hubiera que perseguir un lenguaje de observación neutro y seguro que confiera precisión y certeza a las expresiones de la ciencia, porque tal lenguaje tendría que ser acerca de sensaciones, un lenguaje solipsista, subjetivo, privado. El tipo de lenguaje que proponía Neurath era un lenguaje sobre objetos y sucesos espaciotemporales (lo que entendía por lenguaje "fisicalista") (1931/1983), aunque sus expresiones, incluso las más elementales, los enunciados protocolares, carezcan de la certeza que tanto valoraban otros miembros del Círculo de Viena:

"No hay forma de establecer [...] enunciados protocolares como puntos de partida de las ciencias. No hay ninguna tabula rasa. Somos como los marineros que tienen que reparar su barco en mar abierto, sin poder desmontarlo y reconstruirlo en dique seco" (Neurath, 1932/1983).

En suma, la idea de unificación de la ciencia de Neurath se distancia del proyecto reduccionista de sus compañeros de escuela para situarse en el terreno pragmático de la solución de problemas sociales específicos, cuya complejidad exige la colaboración de distintas ciencias, y se concreta en la elaboración de un compendio organizado de conocimientos científicos teóricos y prácticos que facilite esta colaboración.

\section{VOLVIENDO A LAS DOS CULTURAS}

Por cuanto hemos visto acerca de la unidad de la ciencia, no merece mucha confianza un proyecto reduccionista de la cultura como el sugerido por Wilson. Sin embargo, por otra parte, resulta fácil aplicar a la cuestión de las relaciones entre ciencia y humanidades las ideas de Neurath respecto de la unidad de la ciencia. Los grandes problemas, tanto locales como globales, que afectan actualmente a la humanidad, ya se trate de los movimientos migratorios y de refugiados, el calentamiento global, la creciente desigualdad entre países y dentro de ellos, la corrupción, el terrorismo, etc., no tienen cada uno de ellos una sola causa sino que resultan de la confluencia de causas de diferentes tipos, y hacerles frente con un mínimo de realismo exige abordarlos desde distintos frentes y contar con las aportaciones de profesionales de distintas áreas de nuestra cultura, no solo de la cultura científica y de la tecnología.

Tengamos en cuenta que las soluciones, a corto o largo plazo, de problemas como los mencionados exigen en última instancia decisiones políticas nacionales e internacionales. La lucha contra la desigualdad, por ejemplo, es una cuestión política que parece exigir, entre otras medidas, el establecimiento de un impuesto progresivo sobre el capital a nivel multinacional (Piketti, 2013); y no merecen ninguna confianza medidas contra el calentamiento global que no incluyan políticas que potencien la investigación en nuevas tecnologías no contaminantes y formas de energías renovables lo suficiente abundantes que permitan ir prescindiendo de los hidrocarburos. La importancia de las decisiones políticas multinacionales es aún más obvia ante problemas como los movimientos masivos de refugiados, la corrupción o el terrorismo.

Sin embargo, sería ingenuo esperar que las convicciones personales, igualitarias, ecologistas, humanitarias o de transparencia, de los políticos de turno propicien medidas conducentes a combatir tales problemas. Las disposiciones legislativas y las actuaciones orientadas a tal efecto son o serán, sobre todo, respuestas a las opiniones, sentimientos, presiones generalizadas y movilizaciones de los ciudadanos a favor de ellas. Como la experiencia de los últimos años nos muestra, la inclusión en los programas de los partidos políticos de medidas de "regeneración democrática" o de transparencia no proceden de una súbita 
conversión de nuestros políticos sino de las oleadas de indignación que han producido en la población los múltiples casos de corrupción; y no parece admitir muchas dudas el hecho de que el cumplimiento de los planes presentados por 150 países en la Cumbre sobre el Cambio Climático celebrada en París en diciembre de 2015 para reducir sus propias emisiones de gases dependerá en última instancia de la fuerza que los ciudadanos de los distintos países ejerzan sobre sus gobiernos. En suma, la solución de problemas como los mencionados depende en gran medida de la conciencia que de ellos tengan los ciudadanos, de sus exigencias ante sus gobernantes, de su implicación en las medidas adecuadas que se adopten y de su aceptación de las consecuencias colaterales de estas.

Llegados a este punto es inevitable resaltar la influencia que en la formación de opiniones, creencias, valores, conciencia social y actitudes de los ciudadanos ante los grandes problemas sociales tienen elementos de la cultura como la sociología, la ideología política, la filosofía, la ética, la antropología o la historia: áreas humanísticas cuyos contenidos se difunden y circulan entre la población a través del ensayo, la narrativa, los blogs, las redes sociales, el periodismo de opinión, el cine, la televisión, la radio o el teatro, esto es, medios que, en un país democrático, favorecen la información, la reflexión crítica, la desarticulación de mitos, la discusión racional y la formación de criterios propios en cuestiones para las que la ciencia y la tecnología no siempre aportan respuestas, unas veces porque no han llegado aún a ellas y otras porque sencillamente no forman parte de sus objetivos. Desde esta perspectiva carecen de sentido los enfrentamientos entre distintos aspectos de la cultura, como es el caso de las discusiones y "guerras" entre paladines de la ciencia y de las humanidades. La guerra realmente importante en el ámbito de la cultura es la que se dirige contra los problemas que afligen o amenazan a los ciudadanos y contra las creencias, mitos, tópicos, opiniones y actitudes que dificultan su solución, y en ella es imprescindible la colaboración de distintas áreas de nuestra cultura, sin que en esta tarea ninguna de ellas deba renunciar a su identidad, a su propia perspectiva y a sus objetivos.

Pero finalmente son imprescindibles dos breves comentarios. La conclusión de este artículo es un entendimiento meramente pragmático entre la ciencia y su contexto cultural, una propuesta de mínimos que en modo alguno expresa todo el contenido y la relevancia cultural tanto de la ciencia como de las humanidades. Más allá de sus aplicaciones prácticas, el conocimiento que nos ofrecen las teorías científicas mejor establecidas nos permite encontrar explicaciones de los fenómenos y una imagen de la naturaleza física, biológica y humana mucho más sólida de lo que los distintos relativismos culturales predican y mucho más satisfactoria que las procedentes de creencias pseudocientíficas, revelaciones divinas o antiguas leyendas. Del mismo modo, resultaría injustamente empobrecedor valorar la cultura humanista solo por su capacidad para configurar en los ciudadanos determinadas actitudes o valores orientados a la solución de problemas prácticos comunes. Ello supondría olvidar todos los aspectos éticos y estéticos de las humanidades y, sobre todo, su calidad de ámbito donde se replantean nuestros grandes y viejos problemas y se generan concepciones generales del mundo y de la vida humana que, en consonancia con la ciencia, contribuyen a dar sentido a nuestra existencia.

\section{NOTAS}

1. Las citas de Lutero, Melanchton y Calvino están tomadas de White, 1896, pp. 126-127.

2. Pico de la Mirandola nos presenta a Dios diciendo a Adán: "La naturaleza de las demás criaturas está definida y limitada por las leyes que he establecido. Sin embargo, tú, libre de tales restricciones, podrás trazar por ti mismo los rasgos de tu propia naturaleza [...] No te he hecho celeste ni terrestre, mortal ni inmortal, para que, por ti mismo, como libre y soberano artífice, te forjes de la forma que hayas escogido" (Garin, 1994, pp. 8-9).

3. Como resaltaba Snow (1964/1977), muchos británicos que consideraban imperdonable culturalmente no haber leído alguna obra de Shakespeare no creían tan grave desconocer el contenido del segundo principio de la termodinámica (p. 24).

4. Tampoco Rorty (1988), discutiendo si la ciencia configura una clase "natu- ral" en el contexto de la cultura, creía ver ninguna diferencia esencial entre la química y la crítica literaria o la biología y la moralidad.

5. Cabe destacar en este aspecto las críticas al relativismo de los filósofos Laudan (1996) y Boghossian (2006).

6. Neurath participó activamente en política: trabajó en 1919 en la oficina central de planificación de la breve República Bávara y colaboró con el gobierno austriaco entre 1921 y 1934. 


\section{BIBLIOGRAFÍA}

Aronowitz, S. (1988). Science as Power: Discourse and Ideology in Modern Society. Minneapolis, MN: University of Minnesota Press.

Barnes, B. y Bloor, D. (1982). Relativism, Rationalism and the Sociology of Knowledge. En: Hollis, M. y Lukes, S. (eds.). Rationality and Relativism. Oxford: Blackwell, pp. 21-47.

Boghossian, P. (2006). Fear of Knowledge: Against Relativism and Constructivism. Oxford: Clarendon Press. https://doi.org/10.1093/acprof:o so/9780199287185.001.0001

Brockman, J. (ed.). (1995). The Third Culture. New York: Touchtone.

Capelli, L. M. (ed.) (1906) Petrarca, F. Le traité De sui ipsius et multorum ignorantia. Paris: Librairie Honoré Champion.

Carnap, R. (1928). Der Logische Aufbau der Welt. Leipzig: Felix Meiner Verlag.

Carnap, R., Hahn, H. y Neurath, O. (1929). Wissenschaftliche Weltauffassung: der Wiener Kreis. Wien: Artur Wolf Verlag.

Cartwright, N. (1999). The Dappled World: A Study of the Boundaries of Science. Cambridge: Cambridge University Press. https://doi.org/10.1017/ CB09781139167093

Cartwright, N., Cat, J., Flech, L. y Uebel, T. (1996). Philosophy Between Science and Politics. Cambridge: Cambridge University Press.

Cohen, R. S. y Neurath, M. (eds.) (1983). Philosophical Papers 1913-1946. Dordrecht: Reidel.

Feyerabend, P. K. (1975/1981). Against Method. London: New Left Books. [Trad. esp.: Ribes, D. Tratado contra el método. Madrid: Tecnos].

Garin, E. (ed.) (1994). Pico della Mirandola, G. Oratio de hominis dignitate. Pordedone: Studio Tesi.

Gross, P. R. y Levitt, N. (1994). Higher Superstition: The Academic Left and Its Quarrels With Science. Baltimore: The John Hopkins University Press.

Hacking, I. (1999/2001). The Social Construction of What? Cambridge, MA: Harvard University Press. [Trad. esp.: Sánchez, J. ¿La construcción social de qué? Barcelona: Paidós].
Harding, S. (1986/2010). The Science Question in Feminism. Ithaca: Cornell Universsity Press.

Hawking, S. y Mlodinow, K. (2010). The Grand Design. New York: Randon House. [Trad. esp: Jou, D. El gran diseño. Barcelona: Crítica].

Heidegger, M. (1953/2001). Einführung in die Metaphysik. Tübingen: Niemeyer. [Trad. esp.: Ackermann, A. Introducción a la metafísica. Barcelona: Gedisa].

Irigaray, L. (1987). Sexes et parentés. Paris: Éditions de Minuit.

Latour, B. (1987). Science in Action. Cambridge, MA: Harvard University Press.

Latour, B. (1999). Pandora's Hope. Essays on the Reality of Science Studies. Cambridge, MA: Harvard University Press.

Latour, B. y Woolgar, S. (1979). Laboratory Life. Beverly Hills: Sage.

Laudan, L. (1996). Beyond Positivism and Relativism. Theory, Method and Evidence. Boulder, CO: Westview Press.

Neurath, O. (1930/1983). Ways of the Scientific World Conception. En: Cohen, R. S. y Neurath, M. (eds.). Philosophical Papers 1913-1946. Dordrecht: Reidel, pp. 32-47. https://doi.org/10.1007/978-94009-6995-7_3

Neurath, O. (1931/1973). Empirical Sociology. En: Cohen, R. S. y Neurath, M. (eds.). Empiricism and Sociology. Dordrecht: Reidel, pp. 319-421.

Neurath, O. (1931/1983). Sociology in the Framework of Physicalism. En: Cohen, R. S. y Neurath, M. (eds.). Philosophical Papers 1913-1946. Dordrecht: Reidel, pp. 58-90. https://doi. org/10.1007/978-94-009-6995-7_6

Neurath, O. (1932/1983). Protocol Statements. En: Cohen, R. S. y Neurath, M. (eds.). Philosophical Papers 1913-1946. Dordrecht: Reidel, pp. 91-99. https://doi. org/10.1007/978-94-009-6995-7_7

Neurath, O. (1936/1983). Encyclopedia as Model. En: Cohen, R. S. y Neurath, M. (eds.). Philosophical Papers 1913-1946. Dordrecht: Reidel, pp.145-158.

Neurath, O. (1946/1983). The Orchestration of the Sciences by the Enciclopedism of Logical Empiricism. En: Cohen, R. S. y Neurath, M. (eds.). Philosophical Papers 19131946. Dordrecht: Reidel, pp. 230-242.
Nietzsche, F. (1873/1980). Über Wahrheit und Lüge im aussermoralischen Sinne. [Trad. esp.: Valdés, L. M. y Orduña, T. Sobre verdad y mentira en sentido extramoral. Valencia: Cuadernos Teorema].

Piketti, T. (2013). Le capital au XXle siècle. Paris: Seuil.

Rorty, R. (1988). Is Science a Natural Kind? En: McMullin, E. (ed.). Construction and Constraint. The Shaping of Scientific Rationality. Notre Dame: University of Notre Dame Press, pp. 49-74.

Ross, A. (1996). Science Wars. Introduction. Social Text, 46/47, pp. 1-14.

Snow, C. P. (1964/1977). The Two Cultures and A Second Look. Cambridge: Cambridge University Press. [Trad. esp.: Masó, S. Las dos culturas y un segundo enfoque. Madrid: Alianza].

Sokal, A. (1996). Transgressing the Boundaries: Towards a Transformative Hermeneutics of Quantum Gravity. Science Wars. Social Text, 46/47, pp. 217-252. https://doi.org/10.2307/466856

White, A. D. (1896). A history of the warfare of science with theology in christendom. New York: Appleton.

Wilson, E. W. (1999). Consilience. The Unity of Knowledge. New York: Vintage Books. [Trad. esp.: Ros, J. Consilience. La unidad del conocimiento. Barcelona: Galaxia Gutenberg, 1999]. 